\title{
Reduced expression of DACT2 promotes hepatocellular carcinoma progression: involvement of methylation-mediated gene silencing
}

Sheng Gao ${ }^{1,2 \dagger}$, Zhe Yang ${ }^{1,2 \dagger}$, Zhi-Yun Zheng ${ }^{1,2}$, Jia Yao ${ }^{1,2}$, Feng Zhang ${ }^{1,2,3}$, Li-Ming Wu ${ }^{1,2,3}$, Hai-Yang Xie ${ }^{1,2,3}$, Lin zhou ${ }^{1,2,3}$ and Shu-Sen Zheng ${ }^{1,2,3,4^{*}}$

\begin{abstract}
Background: Hepatocellular carcinoma (HCC) is one of the most aggressive malignancies in humans, and its prognosis is generally poor even after surgery. Many advances have been made to understand the pathogenesis of HCC; however, the molecular mechanisms that lead to hepatocarcinogenesis and progression are still not clearly understood.
\end{abstract}

Methods: The expression of DACT2 in specimens from 30 paired HCCs and an additional 61 HCC patients after liver transplantation was evaluated by quantitative RT-PCR and immunohistochemical analysis. We investigated the methylation status of the DACT2 promoter region. We also analyzed the alterations of the cell cycle, migration and invasion after DACT2 knockdown.

Results: The expression level of DACT2 was significantly lower in HCC tissues than in non-cancerous tissues. Reduced DACT2 expression was associated with large tumor size. DACT2 transcripts were at low levels in hypermethylated liver cancer cells and were restored by exposure to a demethylating agent. Reduced expression of DACT2 in MHCC97L cells induced G1/S arrest, increased cell proliferation, and promoted cell invasion.

Conclusions: Our study suggests that DACT2 is silenced by promoter hypermethylation, and reduced DACT2 can promote liver cancer progression. DACT2 may serve as a novel tumor suppressor gene in HCC.

Keywords: DACT2, Hepatocellular carcinoma, Methylation, Progression

\section{Background}

Primary liver cancer, predominantly consisting of hepatocellular carcinoma (HCC), is the fifth most common cancer and the third most common cause of cancer-related deaths worldwide. It is estimated that approximately 626,000 new cases of HCC occur annually and nearly as many deaths $[1,2]$. Cases in China account for more than $50 \%$ of the world's cases because of the high prevalence of chronic hepatitis B virus infection and liver cirrhosis $[3,4]$.

\footnotetext{
* Correspondence: zyzss@zju.edu.cn

${ }^{\dagger}$ Equal contributors

'Key Laboratory of Combined Multi-organ Transplantation, Ministry of Public

Health, Hangzhou, China

${ }^{2}$ Key Laboratory of Organ Transplantation, Zhejiang Province, Hangzhou,

China

Full list of author information is available at the end of the article
}

HCC is one of the most aggressive malignancies in humans, and its prognosis is generally poor even after surgery [5-7]. Many advances have been made to understand the pathogenesis of HCC; however, the molecular mechanisms leading to hepatocarcinogenesis and progression are still not clearly understood. Therefore, we are still lacking effective therapies of HCC. For better diagnosis and treatment of HCC, it is crucial to identify the genes responsible for the initiation, promotion, and progression of the disease. A detailed understanding of the molecular mechanisms associated with HCC ultimately could improve our current treatment modalities of this disease. HCC is a highly aggressive cancer with activation of multiple signal transduction pathways and various gene alterations [8-10]. The Wnt signaling pathway has been shown to be

\section{Biomed Central}

(c) 2013 Gao et al.; licensee BioMed Central Ltd. This is an Open Access article distributed under the terms of the Creative Commons Attribution License (http://creativecommons.org/licenses/by/2.0), which permits unrestricted use, distribution, and reproduction in any medium, provided the original work is properly cited. 
involved in cell proliferation, apoptosis, differentiation and motility [11]. This pathway is deregulated in a number of cancers, including HCC [12]. Dapper, antagonist of betacatenin, homolog 2 (DACT2), located on chromosome 6q27, belongs to the DACT (Dpr/Frodo) gene family. This family consists of DATC1, 2 and 3 [13,14]. Members of the DACT (Dpr/Frodo) gene family have been shown to modulate $W n t / \beta$-catenin signaling by interacting with Dishevelled (Dvl), a central component of Wnt signaling [15-17]. A number of studies have revealed the biological properties of DACT proteins in human cancer. For example, Yau et al. demonstrated that the expression of HDPR1, the human homolog of Dpr, was frequently and significantly downregulated in HCC. Downregulation of HDPR1 frequently occurs through hypermethylation of the promoter region and allelic loss of the locus [18]. Jiang et al. identified DACT3 as an important negative regulator of $\mathrm{Wnt} / \beta$-catenin signaling and functions as a potential tumor suppressor in colorectal cancer. The expression of DACT3 is consistently suppressed in colon cancer, and its expression is silenced by bivalent histone modification [19]. DACT2 has been described and studied in both fish and mammals $[14,20,21]$. Although there have been relatively few reports about DACT2 in cancer research, the expression level of DACT2 appears to be reduced in some colorectal tumors. To our knowledge, there have been no studies investigating a possible correlation among the expression of DACT2, the clinicopathological factors in HCC, and the relevance of DACT2 expression to oncogenesis.

In this study, we explored DACT2 gene expression in $\mathrm{HCC}$ and its possible association with clinicopathological factors. In addition, the epigenetic regulation of DACT2 in HCC cells was also analyzed.

\section{Methods}

\section{Study population and tissue samples}

Sixty-one HCC patients who had undergone liver transplantation during 2003 and 2005 at our hospital (First Affiliated Hospital, Zhejiang University School of Medicine, Zhejiang, China) were enrolled in this study. The study was approved by the local ethics committee, and informed consent was obtained from all of the patients. Of these patients, HCC was diagnosed either before or after transplantation, which was confirmed by histopathological examination. Complete clinical and laboratory data were available before operation and during follow-up, and all patients were HBV-positive $\left(\mathrm{HBsAg}^{+}\right)$. Distribution of clinicopathological data in the study cohort is given in Table 1. An additional 30 patients with $\mathrm{HCC}$ were also included in the present study. Specimens of cancer tissues and adjacent noncancerous tissues were collected from these patients after obtaining informed consent.
Table 1 Characteristics of the 61 patients and relationship of DACT2 expression with clinicopathological parameters in $\mathrm{HCC}$

\begin{tabular}{|c|c|c|c|}
\hline \multirow[t]{2}{*}{ Variables } & \multicolumn{3}{|c|}{ DACT2 expression } \\
\hline & Low $(n=31)$ & High $(n=30)$ & $P$ value* \\
\hline \multicolumn{4}{|l|}{ Age (years) } \\
\hline$\leq 50$ & 17 & 18 & 0.797 \\
\hline$>50$ & 14 & 12 & \\
\hline \multicolumn{4}{|l|}{ Gender } \\
\hline Female & 1 & 4 & 0.195 \\
\hline Male & 30 & 26 & \\
\hline \multicolumn{4}{|l|}{ PVTT } \\
\hline Negative & 21 & 19 & 0.791 \\
\hline Positive & 10 & 11 & \\
\hline \multicolumn{4}{|c|}{ Preoperative AFP level (ng/ml) } \\
\hline$\leq 400$ & 15 & 18 & 0.444 \\
\hline$>400$ & 16 & 12 & \\
\hline \multicolumn{4}{|c|}{ Histopathologic grading } \\
\hline Well + moderate & 24 & 19 & 0.270 \\
\hline Poor & 7 & 11 & \\
\hline \multicolumn{4}{|l|}{ Tumor size (cm) } \\
\hline$\leq 5$ & 9 & 17 & 0.040 \\
\hline$>5$ & 22 & 13 & \\
\hline \multicolumn{4}{|l|}{ Tumor number } \\
\hline Single & 10 & 10 & 1.000 \\
\hline Multiple & 21 & 20 & \\
\hline
\end{tabular}

PVTT, portal vein tumor thrombi; AFP, alpha-fetoprotein. ${ }^{*}$ Chi-square test (Fisher's exact test).

\section{Reverse-transcription PCR and real-time reverse-transcription} PCR

Total RNA was extracted from hepatic specimens or cell lines, and cDNA was synthesized from total RNA. The messenger RNA (mRNA) expression level of theDACT2 was determined by RT-PCR. The polymerase chain reaction (PCR) primer sequences for the DACT2 mRNA amplification have been described previously [19]. Realtime PCR reactions were performed by the ABI7500 system (Applied Biosystems, CA, USA) and SYBR green dye (Applied Biosystems).

\section{Immunohistochemistry}

Immunohistochemical studies of DACT2 were performed on surgical specimens from HCC patients with cancerous and noncancerous adjacent tissues. Primary rabbit polyclonal antibodies against DACT2 (ProSci Inc., CA, USA) were used at a dilution of 1:600. Immunohistochemistry was performed as described previously [22].

\section{Cell lines}

Liver cancer cell lines SMMC-7721, HepG2 and Hep3B as well as the metastasis-capable human HCC cell lines 
MHCC97L (Shanghai Institute of Cell Biology, Shanghai, China) and HCCLM3 (Liver Cancer Institute of Fudan University, Shanghai, China) were purchased. All cell lines were maintained in the recommended culture condition and incubated at $37^{\circ} \mathrm{C}$ in a humidified environment containing $5 \% \mathrm{CO}_{2}$.

\section{Bisulfite modification of DNA}

Genomic DNA was extracted from cell lines using the QIAamp DNA Mini Kit (Qiagen, Hilden, Germany). DNA samples were modified using EZ DNA Methylation Golden Kit (Zymo Research, CA, USA). Bisulfite converts unmethylated CpG sites to UpG without modifying methylated sites.

\section{Demethylation with the DNA demethylating agent 5-Aza-2'-deoxycytidine}

Liver cancer cells were plated at a density of $5 \times 10^{4}$ cells $/ \mathrm{ml}$. After $24 \mathrm{~h}$, cells were treated with $10 \mu \mathrm{mol} / \mathrm{l}$ of the DNA demethylating agent 5-aza-2'deoxycytidine (5-aza-dC) (Sigma-Aldrich, St. Louis, MO, USA) for $96 \mathrm{~h}$. DNA and RNA were then extracted from cells.

\section{MSP and bisulfite genomic sequencing}

The methylation status of the DACT2 CpG islands was determined by MSP. The primers for detection of the methylated and unmethylated DACT2 CpG islands were described in the previous study [19]. For bisulfite genomic sequencing, $2 \mu \mathrm{l}$ of bisulfite-treated DNA was amplified using primers [19]. The PCR products were cloned into a PUCm-T vector. Three to five colonies were randomly chosen and sequenced for plasmid DNA extraction with QIAprep Spin Miniprep Kit (Qiagen, Hilden, Germany).

\section{RNA interference}

Short interfering RNA (siRNA) duplexes (GenePharma, Shanghai, China) were used to downregulate DACT2 expression, and a nonsilencing siRNA was used as negative control. After growth in the plate for $24 \mathrm{~h}$, cells were transfected with $33 \mathrm{nM}$ siRNA using Lipofectamine 2000 transfection reagent (Invitrogen, CA, USA) according to the manufacturer's instructions. The efficacy of transfection was checked by real-time PCR after $48 \mathrm{~h}$.

\section{Cell cycle analysis}

MHCC97L cells were transfected with DACT2 siRNA or a negative control. After $48 \mathrm{~h}$, cells were harvested and stained with a DNA PREP kit (Beckman Coulter, Fullerton, CA). The percentage of cells in G0/G1, S, and $\mathrm{G} 2 / \mathrm{M}$ phase was quantified using flow cytometry analysis according to the manufacturer's instructions (Cytomics FC 500, Beckman Coulter). Analysis of cell cycle data was performed with Multicycle (Los Angeles,
CA) analysis software. All experiments were completed in triplicate.

\section{Cell invasion and motility assay}

Cell invasion analysis was performed using 24-well transwell plates (Millipore, Billerica, MA). Forty-eight hours after RNA interference, the filters were coated with Matrigel (BD Bioscience, San Jose, CA) in the upper compartment and seeded with $200 \mu \mathrm{l}$ of $0.8 \times 10^{5}$ cells. The lower compartment was filled with cell culture medium supplemented with $15 \%$ fetal bovine serum. After $48 \mathrm{~h}$, migrated cells on the bottom surface were fixed with methanol and stained with $0.1 \%$ Crystal Violet. The cell motility assay was performed similarly, except the cells were applied into the uncoated filter and incubated for $24 \mathrm{~h}$. The invading cells were examined, counted, and photographed using digital microscopy. Three fields were counted per filter in each group.

\section{Statistical analysis}

The relationship between DACT2 expression and clinicopathological variables were assessed by the chi-square test. The detailed statistical tests used in the study are shown in Results and in the table and figure legends. $P<0.05$ was considered statistically significant.

\section{Results}

\section{Analysis of DACT2 expression in clinical samples}

Expression of DACT2 mRNA in HCC tumor specimens was lower than in nontumoral liver specimens in 24 of the 30 paired cases. The average level of DACT2 mRNA expression in HCC tissues was 2.25 -fold lower than in adjacent noncancerous tissues $(4.68 \pm 6.96$ vs. $10.55 \pm$ 14.6; $P<0.05)$ as shown in Figure 1A. DACT2 protein expression was also investigated by immunohistochemistry in tumor tissues and the nontumoral liver counterparts $(n=26)$, as is shown in Figure 1B,C,D. In healthy liver tissues, DACT2 was almost homogeneously expressed in non-neoplastic hepatocytes. In tumor tissues, two staining patterns could be distinguished. In most patients (21/26 cases), DACT2 expression was markedly decreased in tumor tissues compared with nontumorous liver tissues. Only five samples showed comparable expression levels of DACT2 between tumor and noncancerous liver specimens.

\section{Correlation of DACT2 expression with clinicopathological parameters}

In this study, we also included another cohort of 61 HCC patients who had undergone liver transplantation. We divided them into two expression groups. The low-expression group (30 samples) included patients whose tumor tissue expressed DACT2 mRNA below the median level. The high-expression group 


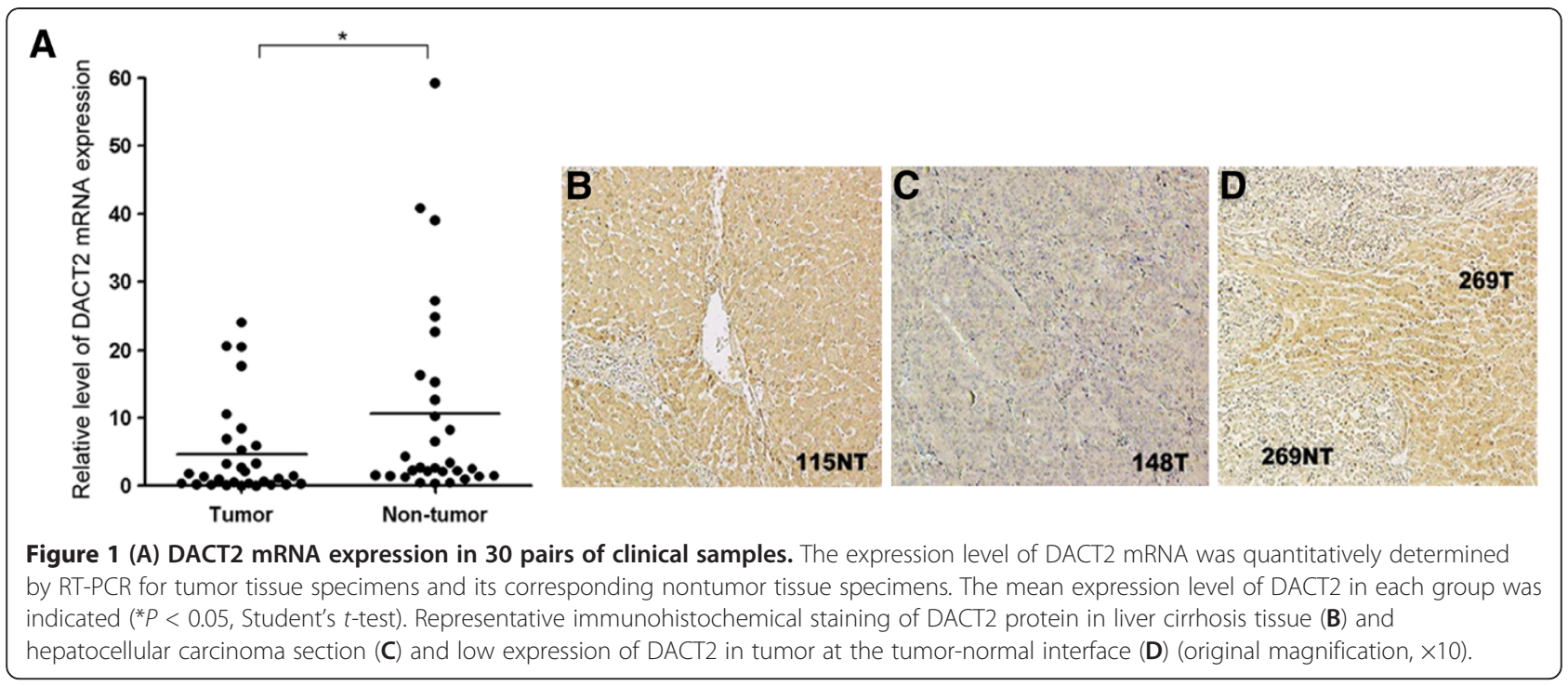

$(n=31)$ consisted of patients whose tumor tissue expressed DACT2 mRNA above the median value. Clinicopathological parameters are listed in Table 1 in relation to DACT2 mRNA expression status. In the relationship between DACT2 expression and the clinicopathological data, we found that DACT2 expression was negatively correlated with tumor size $(P=0.04)$. Tumors with low DACT2 expression were significantly larger in size $(>5 \mathrm{~cm})$ than those with high expression. There was no statistically significant correlation between DACT2 expression and age, gender, AFP, histopathological grading, or tumor number.

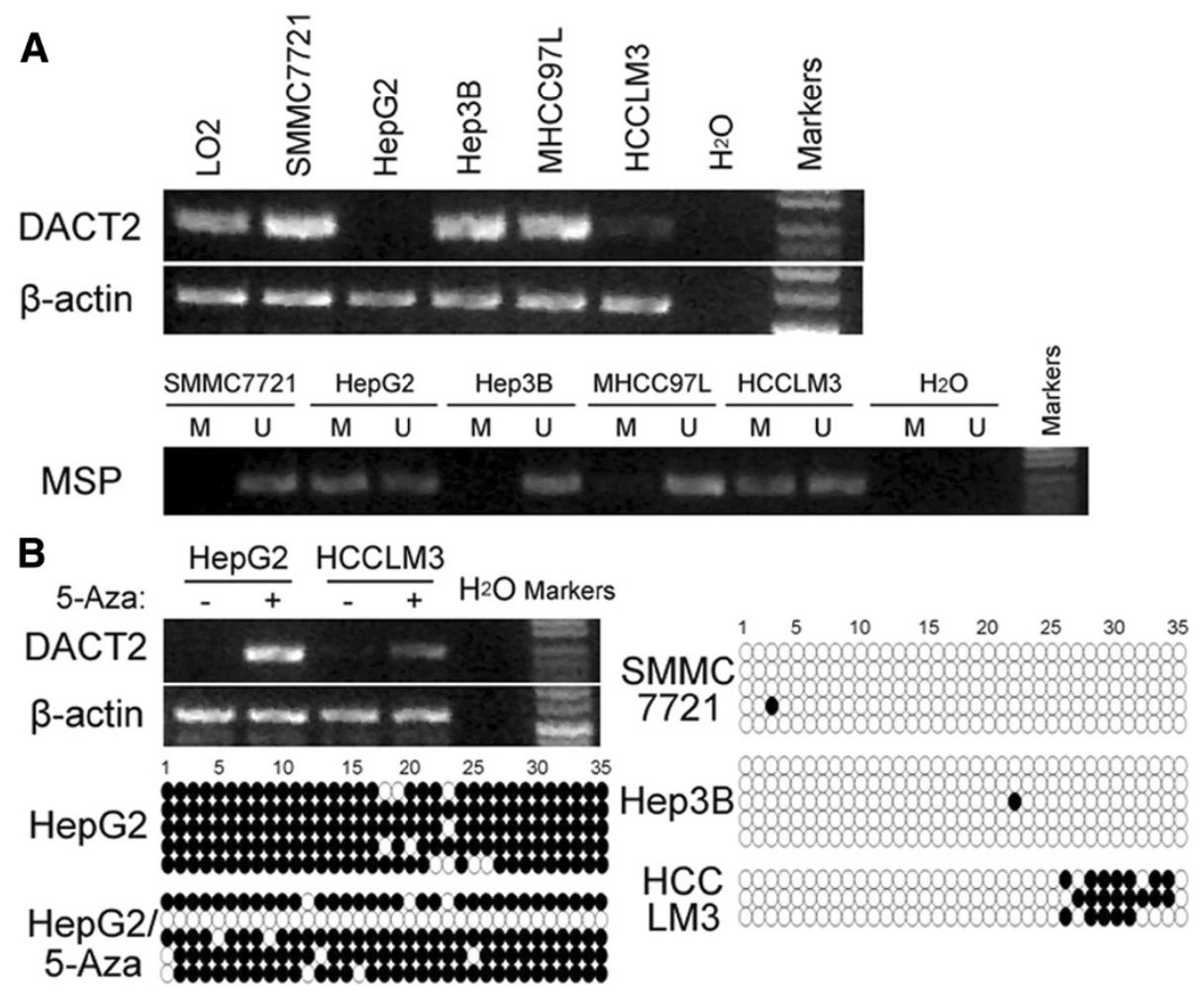

Figure 2 (A) DACT2 mRNA expression and its methylation status in liver cancer cell lines. (B) The mRNA expression of DACT2 was restored after treatment with 5-Aza. Demethylation was observed in the HepG2 cell line after treatment with 5-Aza, and the methylation of DACT2 in several liver cancer cell lines was examined by bisulfite genomic sequencing. 


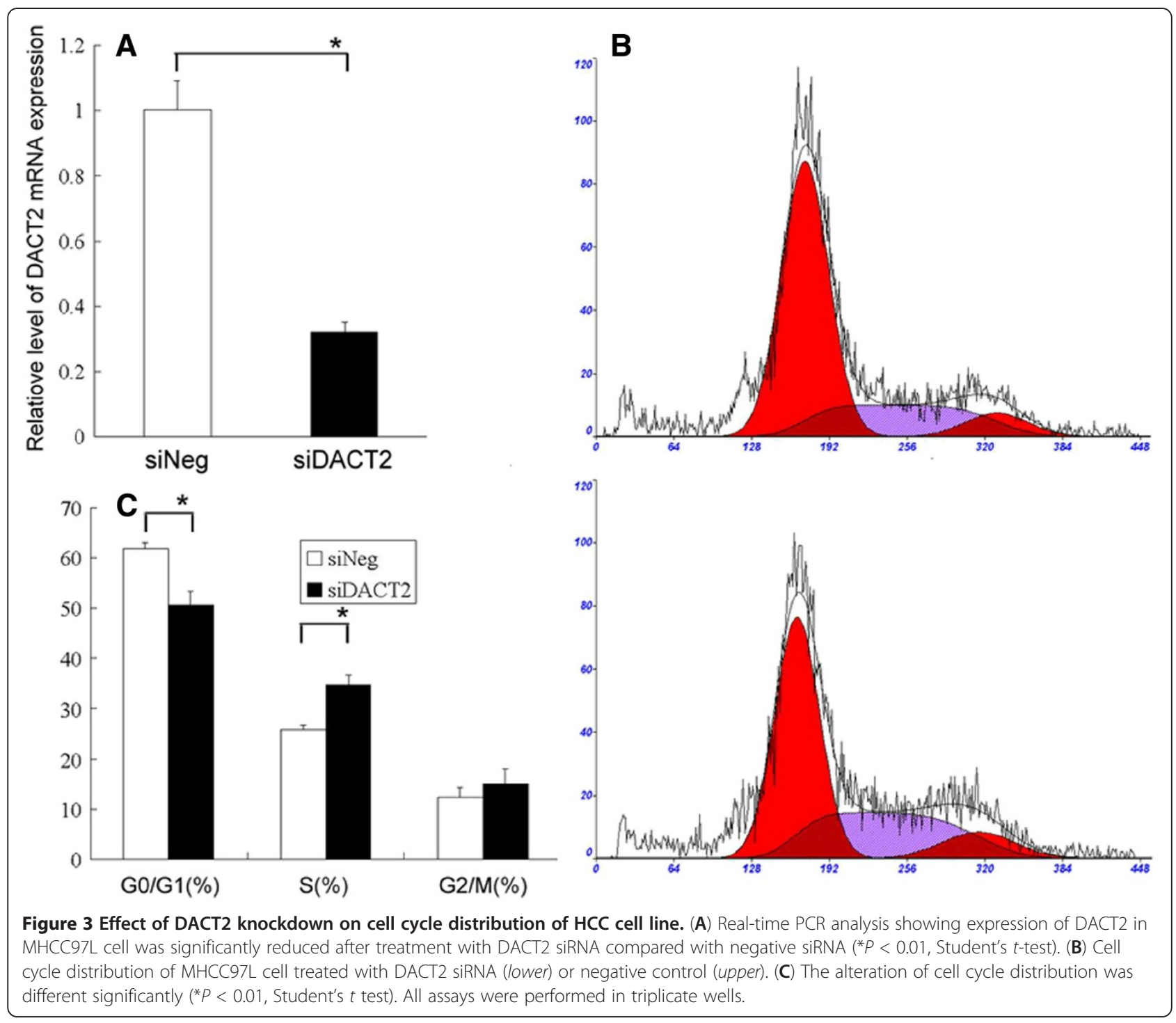

\section{Downregulation and promoter methylation of DACT2 in} liver cancer cells

We first detected the expression level of DACT2 in five liver cancer cell lines. The results showed that DACT2 transcript was silenced or reduced in HepG2 and HCCLM3 cells (Figure 2A). To examine the role of promoter methylation in the silencing of DACT2, we further analyzed the methylation status of the DACT2 gene; the results indicate that the DACT2 gene promoter was partially methylated in cell lines with reduced or silenced expression (Figure 2A). To demonstrate whether methylation mediates DACT2 silencing, we treated two methylated cell lines that showed silencing of DACT2 with 5-Aza. 5-Aza restored DACT2 expression in both cell lines with obvious demethylation observed in the treated HepG2 cell line by bisulfite genomic sequencing (Figure 2B), suggesting that DNA methylation plays a crucial role in the transcriptional silencing of DACT2 in liver cancer cells.
Cell cycle alterations after DACT2 silencing

As shown in Figure 3A, the inhibitory efficiency of siRNAs for gene transcription was significant in morderatemetastasic potential MHCC97L cells. To determine the role of DACT2 in tumor cell cycle progression and tumor cell proliferation, we examined the effect of DACT2 knockdown in MHCC97L cells on the cell cycle. After $48 \mathrm{~h}$ of transfection, cell proliferation as determined by the number of cells in the $S$ phase was increased from $25.9 \%$ to $34.6 \%$ $(P<0.01)$, as is shown in Figure 3B,C. Moreover, the number of cells in the G0/G1 phase decreased from $61.7 \%$ to 50.5\% $(P<0.01)$.

\section{DACT2 silencing promotes cell migration and invasion}

The effect of DACT2 on the migration and invasiveness of HCC cells was analyzed using the Matrigel model. We found that the average number of migratory and invaded cells transfected with DACT2 siRNA was significantly 


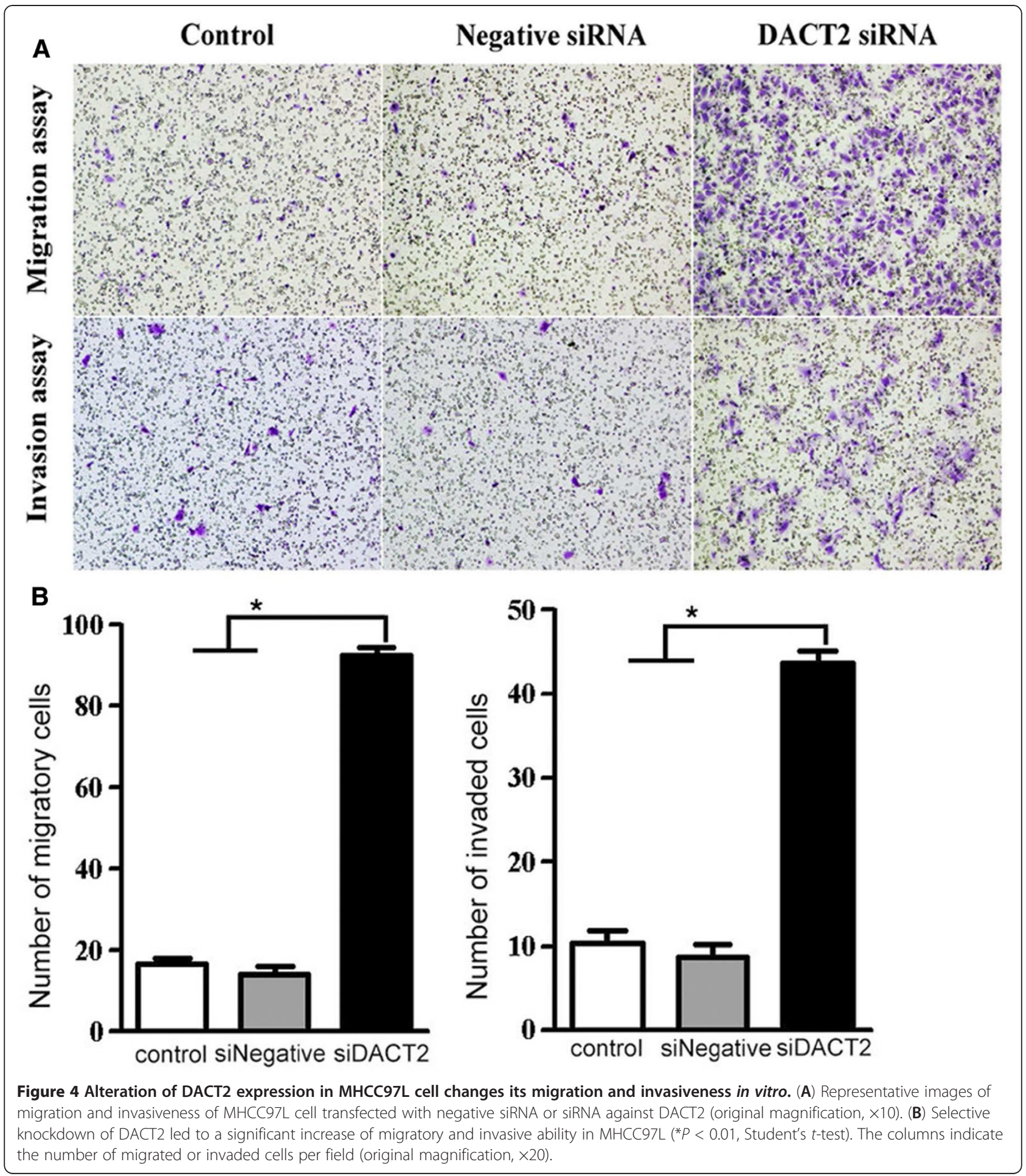

increased when compared to those with negative control siRNA (Figure 4), indicating that the invasive potential of MHCC97L cells increased after DACT2 knockdown. This suggests that DACT2 also influences the migration and invasiveness of HCC cells.

\section{Discussion}

In the current study, we found that the expression level of DACT2 was downregulated in human hepatocellular carcinoma by quantitative RT-PCR and immunohistochemical analysis. Similarly, in a previous study, Jiang 
et al. also found that DACT2 expression is also reduced in some colorectal tumors [19]. Therefore, DACT2 was implicated as a tumor suppressor gene in some types of human cancers. We further speculate about whether the expression level of DACT2 might be associated with clinicopathological parameters in liver transplantation HCC patients. Clinicopathological analysis revealed that HCC patients with low DACT2 expression was correlated with a larger tumor size $(>5 \mathrm{~cm})$ than those with high DACT2 expression. DACT2 may thus play a critical role in the proliferation and progression of HCC cells. To the best of our knowledge, there have been no prior reports studying the associations between DACT2 gene expression and clinicopathological parameters in human cancer. Thus, this is the first study to determine the expression pattern of DACT2 and report the clinical significance of DACT2 gene expression in HCC. Because of the possible role of DACT2 in tumor suppression and its reduced expression in cancer, the correlation of DACT2 expression and its methylation status have been characterized in colorectal cancer. Aberrant promoter hypermethylation of DACT2 was detected in several tumor samples with reduced DACT2 expression [19]. This finding led us to investigate the potential correlation of expression level and promoter methylation of DACT2 in HCC. Similar to the previous report on colon cancer cells, we found that DACT2 expression was present at very low or undetectable levels in some HCC cell lines and that 5-Aza-dC restored the transcription level of DACT2 (Figure 3). These data suggested that the downregulation of DACT2 expression was closely associated with promoter hypermethylation in vitro. Taken together, these findings further support that DACT2 is inactivated epigenetically in a number of human solid tumors including HCC and that promoter hypermethylation may be a critical mechanism for the transcriptional silencing of the DACT2 gene in liver cancer cell lines.

The tumor-suppressive function of DACT2 in HCC was investigated further by in vitro assays. In DACT2 expression silenced MHCC97L cells, FACS analysis revealed a significant increase of $S$ phase cells and a decrease of G0/G1 phase cells, indicating G1/S arrest of the cell cycle and a significant promotion of cell proliferation. In addition, DACT2 knockdown in MHCC97L cells also resulted in increased ability of tumor cell invasion and metastasis (Figure 4). Taken together, these results suggest the role of DACT2 as a functional tumor suppressor gene through suppressing tumor cell proliferation, migration and invasion in HCC.

Our studies are the first to clearly identified the biological functions of DACT2 in human HCC cells; however, the molecular basis for how DACT2 knockdown leads to increased proliferation and invasion in HCC is still unknown. A number of studies have tried to illuminate the molecular mechanisms of the Dapper family proteins in regulating cell behavior. Waxman et al. found that zebrafish Dpr2 is a vital regulator of the noncanonical Wnt/Ca2+-PCP pathway [23]; however, $\mathrm{Su}$ et al. suggested that mouse Dpr2 inhibits the Wnt/ JNK pathway and functions as a negative regulator of the TGF- $\beta$ /Nodal signal pathway [24]. Thus, the Dpr2 protein may function to regulate distinct cell signaling pathways in a context-dependent manner. Therefore, the molecular mechanisms by which DACT2 exerts the tumor-suppressor effect in HCC needs further study.

It should be noted that there were some limitations in the current study. HCC is a tumor of diverse etiology, and our study population predominantly consisted of patients with hepatitis B-induced HCC. It is therefore important to determine whether DACT2 still functions as a tumor suppressor gene in patients with other underlying liver diseases. In addition, the study was confined to the Han Chinese population, which should be taken into account when evaluating the potential applicability of our findings to other ethnic populations. Lastly, the sample size was small because of the strict eligibility of the study population. The study should therefore be viewed as hypothesis generating, and our findings should be confirmed by examining larger clinical samples.

\section{Conclusion}

We demonstrate that expression of DACT2 is downregulated in HCC compared to adjacent healthy liver tissues and that reduced DACT2 expression is significantly correlated with large tumor size. Furthermore, promoter hypermethylation is the principal regulatory mechanism of DACT2 inactivation in HCC cells. Functionally, DACT2 is an important tumor suppressor gene with key roles of regulating tumor cell proliferation, migration and invasion in the development and progression of HCC. Our study suggests that DACT2, which was silenced by promoter hypermethylation, may serve as a novel candidate tumor suppressor gene in HCC.

\section{Competing interests \\ The authors declare that they have no competing interests.}

\section{Authors' contributions}

SSZ and LZ co-conceived the study and SSZ led the study. LZ, ZY and SG planned and executed experiments and analyses, supervised data acquisition, performed bioinformatic analyses, and carried out the experiments. LMW and JY provided pathological validation and technical support. ZYZ, FZ and LMW collected clinical samples. ZY, HYX and SG provided valuable input regarding study design, data analysis, and interpretation of results. ZY and SG wrote the manuscript. All authors read and approved the final manuscript.

\section{Acknowledgments}

This study was supported by the National S\&T Major Project (no. 2008ZX10002-026) and National Key Technology R\&D Program (no. 2008BAl60B03). The authors thank the study participants, the many surgeons 
and nurses who kindly facilitated in the recruitment and collection of patient information. We thank Xiao-wen Feng for technological assistance.

\section{Author details}

'Key Laboratory of Combined Multi-organ Transplantation, Ministry of Public Health, Hangzhou, China. ${ }^{2}$ Key Laboratory of Organ Transplantation, Zhejiang Province, Hangzhou, China. ${ }^{3}$ Division of Hepatobiliary and Pancreatic Surgery, Department of Surgery, First Affiliated Hospital, Zhejiang University School of Medicine, Hangzhou, China. ${ }^{4}$ Key Laboratory of Combined Multi-organ Transplantation, Ministry of Public Health, the First Affiliated Hospital, Zhejiang University School of Medicine, 79 Qingchun Road, Hangzhou 310003, People's Republic of China.

Received: 21 October 2012 Accepted: 16 February 2013

Published: 7 March 2013

\section{References}

1. Parkin DM: Global cancer statistics in the year 2000. Lancet Oncol 2001, 2:533-543.

2. Parkin DM, Bray F, Ferlay J, Pisani P: Global cancer statistics, 2002. CA Cancer J Clin 2005, 55:74-108.

3. El-Serag HB, Rudolph KL: Hepatocellular carcinoma: epidemiology and molecular carcinogenesis. Gastroenterology 2007, 132:2557-2576.

4. Yuen MF, Hou JL, Chutaputti A: Hepatocellular carcinoma in the Asia Pacific region. J Gastroenterol Hepatol 2009, 24:346-353.

5. Lo CM, Fan ST: Liver transplantation for hepatocellular carcinoma. Br J Surg 2004, 91:131-133.

6. Thomas MB, Zhu AX: Hepatocellular carcinoma: the need for progress. J Clin Oncol 2005, 23:2892-2899.

7. Zheng SS, Xu X, Wu J, Chen J, Wang WL, Zhang M, Liang TB, Wu LM: Liver transplantation for hepatocellular carcinoma: Hangzhou experiences. Transplantation 2008, 85:1726-1732.

8. Aravalli RN, Steer CJ, Cressman EN: Molecular mechanisms of hepatocellular carcinoma. Hepatology 2008, 48:2047-2063.

9. Farazi PA, DePinho RA: Hepatocellular carcinoma pathogenesis: from genes to environment. Nat Rev Cancer 2006, 6:674-687.

10. Llovet JM, Bruix J: Molecular targeted therapies in hepatocellular carcinoma. Hepatology 2008, 48:1312-1327.

11. Nelson WJ, Nusse R: Convergence of Wnt, beta-catenin, and cadherin pathways. Science 2004, 303:1483-1487.

12. Herbst A, Kolligs FT: Wnt signaling as a therapeutic target for cancer. Methods Mol Biol 2007, 361:63-91.

13. Fisher DA, Kivimae S, Hoshino J, Suriben R, Martin PM, Baxter N, Cheyette BN: Three Dact gene family members are expressed during embryonic development and in the adult brains of mice. Dev Dyn 2006, 235:2620-2630.

14. Katoh M: Identification and characterization of human DAPPER1 and DAPPER2 genes in silico. Int J Oncol 2003, 22:907-913.

15. Cheyette BN, Waxman JS, Miller JR, Takemaru K, Sheldahl LC, Khlebtsova N Fox EP, Earnest T, Moon RT: Dapper, a Dishevelled-associated antagonist of beta-catenin and JNK signaling, is required for notochord formation. Dev Cell 2002, 2:449-461.

16. Gloy J, Hikasa H, Sokol SY: Frodo interacts with Dishevelled to transduce Wnt signals. Nat Cell Biol 2002, 4:351-357.

17. Zhang L, Gao X, Wen J, Ning Y, Chen YG: Dapper 1 antagonizes Wnt signaling by promoting dishevelled degradation. J Biol Chem 2006, 281:8607-8612.

18. Yau TO, Chan CY, Chan KL, Lee MF, Wong CM, Fan ST, Ng IO: HDPR1, a novel inhibitor of the WNT/beta-catenin signaling, is frequently downregulated in hepatocellular carcinoma: involvement of methylation-mediated gene silencing. Oncogene 2005, 24:1607-1614

19. Jiang X, Tan J, Li J, Kivimae S, Yang X, Zhuang L, Lee PL, Chan MT, Stanton LW, Liu ET, Cheyette BN, Yu Q: DACT3 is an epigenetic regulator of Wnt/ beta-catenin signaling in colorectal cancer and is a therapeutic target of histone modifications. Cancer Cell 2008, 13:529-541.

20. Meng F, Cheng X, Yang L, Hou N, Yang X, Meng A: Accelerated re-epithelialization in Dpr2-deficient mice is associated with enhanced response to TGFbeta signaling. J Cell Sci 2008, 121:2904-2912.

21. Zhang L, Zhou H, Su Y, Sun Z, Zhang H, Zhang Y, Ning Y, Chen YG, Meng A Zebrafish Dpr2 inhibits mesoderm induction by promoting degradation of nodal receptors. Science 2004, 306:114-117.
22. Zhang F, Wu LM, Zhou L, Chen QX, Xie HY, Feng XW, Zheng SS: Predictive value of expression and promoter hypermethylation of XAF1 in hepatitis $B$ virus-associated hepatocellular carcinoma treated with transplantation. Ann Surg Oncol 2008, 15:3494-3502.

23. Waxman JS, Hocking AM, Stoick CL, Moon RT: Zebrafish Dapper1 and Dapper2 play distinct roles in Wnt-mediated developmental processes. Development 2004, 131:5909-5921.

24. Su Y, Zhang L, Gao X, Meng F, Wen J, Zhou H, Meng A, Chen YG: The evolutionally conserved activity of Dapper2 in antagonizing TGF-beta signaling. FASEB J 2007, 21:682-690.

doi:10.1186/1477-7819-11-57

Cite this article as: Gao et al:: Reduced expression of DACT2 promotes hepatocellular carcinoma progression: involvement of methylationmediated gene silencing. World Journal of Surgical Oncology 2013 11:57.

\section{Submit your next manuscript to BioMed Central and take full advantage of:}

- Convenient online submission

- Thorough peer review

- No space constraints or color figure charges

- Immediate publication on acceptance

- Inclusion in PubMed, CAS, Scopus and Google Scholar

- Research which is freely available for redistribution 